\title{
Begriffsbestimmungen und theoretische Ansätze
}

Meine Revision des Qualia-Begriffs erfordert vorgängig eine begriffliche Bestimmung und theoretische Einordnung derjenigen somatischen, emotiven und kognitiven Phänomene, die meiner Arbeitsthese gemäss (neben den meistdiskutierten sinnlichen Qualia) bei einem phänomenal adäquaten Qualia-Begriff nicht ignoriert werden dürfen. Diese betreffen 1.) emotive Erlebnisqualitäten, die man als >Emotion<, >Stimmung< oder >Gefühl< bezeichnet; 2.) die mit emotiven Erlebniszuständen korrelierenden somatoviszeralen Wahrnehmungen bzw. `Empfindungen ‘; 3.) die Interdependenz von bestimmten Modi des Denkens und emotionalem Erleben bzw. emotiv-kognitiven Formen des Erlebens; 4.) die nicht diesen emotiven Denkmodi zurechenbaren Formen des Erlebens von Gedanken. Im Rahmen dieser Arbeit kann ich jedoch an dieser Stelle nur kurz und im Sinne einer Arbeitsdefinition auf diese mannigfaltigen und komplexen emotiv-somatischen, emotiv-kognitiven und kognitiven Kategorien des Erlebens eingehen.

\subsection{Emotionen, Gefühle und Empfindungen}

Prinz und Damasio bezeichnen das subjektiv-phänomenale Erleben emotionaler Zustände als »feelings«, im Gegensatz zu den öffentlich beobachtbaren affektiv-emotionalen Zuständen und damit verbundenen physiologischen Vorgängen, die sie »Emotions « nennen. ${ }^{1}$ So konstatiert Prinz: $»[. .$.$] emotions$ are feelings when conscious, and they are not feelings when unconscious « ${ }^{2}$ Nach dieser Definition sind Emotionen im engeren Sinne also keine Erlebniszustände: »[...] emotions are actions or movements, many of them public, visible to others as they occur in the face, in the voice, in specific behaviours. [...] Emotions play out in the theater of the body. Feelings play out in the theater of the mind $\ll^{3}$ Dennoch sind Emotionen bzw. die mit ihnen verbundenen

1 Vgl. Prinz, Jesse: »Are Emotions Feelings?«, Journal of Consciousness Studies, [2005] Vol. 12, No. 8-10, S. 9 und 23. Vgl. ebenfalls Damasio, Antonio R.: Looking for Spinoza. Joy, sorrow and the feeling brain, London: Vintage 2004, S. 27, 42 und 86.

2 Prinz, Jesse: »Are Emotions Feelings?«, Journal of Consciousness Studies, [2005] Vol. 12, No. 8-10, S. 9 .

3 Damasio, Antonio R.: Looking for Spinoza. Joy, sorrow and the feeling brain, London: Vintage 2004, S. 28. 
somatischen Zustände bei >feelings< involviert. So definiert Damasio >feelings als Zustände des subjektiven Erlebens bestimmter somatischer und kognitiver Zustände: »My hypothesis, then, [...] is that a feeling is the perception of a certain state of the body along with the perception of a certain mode of thinking and of thoughts with certain themes «. ${ }^{4}$

Für den Nachweis der Notwendigkeit einer Revision des Qualia-Begriffs und dessen Neukonzeption sind jedoch die dem emotionalen Erleben zugrundeliegenden physiologischen Vorgänge nur im Hinblick auf ihre Phänomenologie von Relevanz. Aus diesem Grund ist in dieser Arbeit, wenn von >Emotionen< oder >Gefühlen< die Rede ist, immer das Erleben von Emotionen gemeint. Wenn dagegen von den mit emotionalem Erleben im Zusammenhang stehenden physiologischen Vorgängen die Rede ist, werde ich dafür Begriffe verwenden, die direkt auf die oben erwähnten, öffentlich beobachtbaren, körperlichen Zustände verweisen (d.h. also Begriffe wie >emotionsspezifische propriozeptive und viszerale Rückmeldungsmuster<, >somatoviszerale Zustände<, $>$ Rückmeldungsmuster der Gesichtsmuskulatur und des musculoskeletalen Systems< etc.).

Im Zusammenhang mit dem in Abschnitt 8.2.3 thematisierten Erleben emotionaler Zustände bzw. mit dem, was Damasio »feelings« ${ }^{5}$ (bzw. »Gefühle«) nennt, werde ich der Einfachheit halber zumeist von semotionalem Erleben $<$ und nur selten von >Gefühlen $<$ sprechen. Insofern verwende ich in dieser Arbeit >Emotion<, >emotionales Erleben` und `Gefühl synonym, während mit dem Begriff >Empfindung a ausschliesslich auf körperliches Erleben wie somatosensorische ${ }^{6}$ bzw. somatoviszerale ${ }^{7}$ Empfindungen, Schmerzen, Druckoder Wärmeempfindungen Bezug genommen wird.

4 A.a.O., S. 86 [Hervorhebung im Original].

5 Ebd. [Hervorhebung im Original]. Vgl. dazu auch a.a.O., S. 27, 42 und 86.

6 Als somatosensorisch bezeichnet man diejenigen Empfindungen, die durch die Reizung der Sensoren unseres Körpers hervorgerufen werden. Davon ausgenommen sind aber die so genannten »spezifischen« Sinnesorgane, wie Hör-, Seh-, Geruchssinn etc. die im Kopf lokalisiert sind. Vgl. Handwerker, Hermann: »Somatosensorik«, in: Neuro- und Sinnesphysiologie, Schmidt, Robert F. [Hrsg.], Berlin: Springer 1998, S. 221.

7 Die Gesamtheit dieser somatosensorischen Empfindungen wird auch als somatoviszerale Sensibilität bezeichnet. Vgl. dazu a.a.O., S. 221 und 237. 
Stimmungen werden von Emotionen insofern unterschieden, als sie »keine klaren intentionalen Objekte ${ }^{8}{ }^{8}$ haben. ${ }^{9}$ Die Spezifität des Bezuges einer Stimmung gilt gewöhnlich als weniger ausgeprägt als bei Emotionen, sodass das Objekt einer Stimmung »everything ${ }^{10}{ }^{10}$ oder $»$ nothing in particular ${ }^{11}$ sein kann. ${ }^{12}$ Goldie moniert jedoch diesbezüglich, dass eine scharfe Abgrenzung zwischen Emotionen und Stimmungen nicht möglich sei. ${ }^{13}$ Denn Stimmungsbezüge hätten immer auch einen gewissen Grad von Spezifität, wogegen sich Emotionen ebenfalls auf unspezifische Objekte beziehen können. ${ }^{14}$ Auch das Unterscheidungskriterium längerer zeitlicher Erlebnisperioden von Stimmungen gegenüber kürzeren bei Emotionen ${ }^{15}$ kritisiert Goldie mit dem Hinweis, dass zu wenig zwischen Emotionen, die sehr lange andauern können und (kürzeren) emotionalen Episoden unterschieden wird. ${ }^{16}$ Emotionen und Stimmungen haben demnach sehr viel gemeinsam. ${ }^{17}$ Slaby zufolge neigen Emotionen dazu, sich zu Stimmungen zu »entspezifizieren «. ${ }^{18}$ So kann eine Emotion den Fokus mehr oder weniger verlieren und sich, diffus werdend, in eine Stimmung verwandeln: »[...] emotion can blur out of focus into the non-specifity of mood «. ${ }^{19}$ Umgekehrt kann sich nach Goldie eine Stimmung in

8 Sousa, Ronald de / [Übers.: Pape, Helmut]: Die Rationalität des Gefühls, Frankfurt am Main: Suhrkamp [zuerst 1987, engl.]/2009, S. 31.

9 Vgl. dazu auch Goldie, Peter: The emotions. A philosophical exploration, New York: Oxford University Press 2002, S. 8. Vgl. ebenfalls Stocker, Michael / Hegeman, Elizabeth: Valuing emotions, Cambridge, Massachusetts: Cambridge University Press 2001, S. 22.

10 Goldie, Peter: The emotions. A philosophical exploration, New York: Oxford University Press 2002, S. 143, Fussnote 1.

11 Ebd.

12 Vgl. dazu auch Sousa, Ronald de: »Emotion«, in: A companion to the philosophy of mind, Guttenplan, Samuel [Hrsg.], Malden, Massachusetts: Blackwell 2005, S. 274.

13 Vgl. Goldie, Peter: The emotions. A philosophical exploration, New York: Oxford University Press 2002, S. 143.

14 Vgl. ebd.

15 Dies konstatiert z.B.Ciompi, Luc: Die emotionalen Grundlagen des Denkens. Entwurf einer fraktalen Affektlogik, Göttingen: Vandenhoeck \& Ruprecht 1997, S. 63. Vgl. auch Demmerling, Christoph / Landweer, Hilge: Philosophie der Gefühle. Von Achtung bis Zorn, Stuttgart: Metzler 2007, S. 5 .

16 Vgl. Goldie, Peter: The emotions. A philosophical exploration, New York: Oxford University Press 2002, S. 143.

17 Vgl. ebd.

18 Slaby, Jan: Gefühl und Weltbezug. Die menschliche Affektivität im Kontext einer neoexistentialistischen Konzeption von Personalität, Paderborn: Mentis 20o8, S. 166.

19 Goldie, Peter: The emotions. A philosophical exploration, New York: Oxford University Press 2002, S. 148. 
einer Emotion manifestieren bzw. fokussieren. ${ }^{20}$ Die Unterschiede zwischen Emotion und Stimmung, wie z.B. die Spezifität ihres Bezuges, sind daher eher gradueller Natur. ${ }^{21}$ Im Hinblick auf die in dieser Arbeit auszuweisende Phänomenalität von Stimmungen und Emotionen, genügt es jedoch an dieser Stelle darauf hinzuweisen, dass Stimmungen vorwiegend im Abschnitt 8.2.4 (Phänomenalität emotiv-kognitiven Erlebens) einbezogen werden, während die Phänomenalität von Emotionen vor allem im Abschnitt 8.2.3 (emotivphänomenales Erleben) behandelt wird.

\subsection{Emotive und nicht-emotive Formen des Denken}

Für eine analytische Arbeitsdefinition ${ }^{22}$ von $>$ Gedanken sind dessen zentrale Charakteristika der Propositionalität bzw. propositionaler Einstellungen relevant. ${ }^{23}$ Nach Soames sind Propositionen intrinsisch verbunden mit kognitiven Handlungen ${ }^{24}$ und insofern auch Gehalte intentional-kognitiver Zustände. ${ }^{25}$ Kognitive bzw. intentionale Zustände haben eine Beziehung untereinander und zur Welt. Propositionen können als abstrakte Objekte (vergleichbar mit Zahlen in der Physik) verstanden werden, um diese Beziehungen zu beschreiben ${ }^{26}$ und können deshalb u.a. mit dem verglichen werden, was man >Gedanken< nennt. ${ }^{27}$ Eine Proposition zu denken, bedeutet etwas als etwas

\section{Vgl. ebd.}

21 Zur Unterscheidung zwischen Stimmungen und Emotionen vgl. auch: Sousa, Ronald de / [Übers.: Pape, Helmut]: Die Rationalität des Gefühls, Frankfurt am Main: Suhrkamp [zuerst 1987, engl.]/2009, S. 31. Vgl. ebenfalls Ciompi, Luc: Die emotionalen Grundlagen des Denkens. Entwurf einer fraktalen Affektlogik, Göttingen: Vandenhoeck \& Ruprecht 1997, S. 68.

22 Unter einer >analytischen Arbeitsdefinition` verstehe ich eine Definitionsweise im Sinne der analytischen Philosophie.

23 Vgl. dazu Guttenplan, Samuel: »Proposition «, in: A companion to the philosophy of mind, Guttenplan, Samuel [Hrsg.], Malden, Massachusetts: Blackwell 2005, S. 486-487. Vgl. auch Schildknecht, Christiane: Aspekte des Nichtpropositionalen, Bonn: Bouvier 1999, S. 5-6.

24 Vgl. Soames, Scott: »Propositions«, in: The Routledge companion to philosophy of language, Russell, Gillian / Fara, Delia Graff [Hrsg.], New York: Routledge \& Kegan Paul 2012, S. 218.

25 Vgl. a.a.O., S. 213.

26 Vgl. a.a.O., S. 219.

27 Die Diskussionlage darüber, was Propositionen sind, ist jedoch kontrovers. Vgl. auch Schildknecht, Christiane: Aspekte des Nichtpropositionalen, Bonn: Bouvier 1999, S. 5 . Auch die Konzeption, dass Propositionen oder propositionale Einstellungen als mentale Zustände betrachtet werden können, ist nicht unumstritten. So können für McGrath 
zu prädizieren: wenn eine Person bspw. die Proposition denkt, dass eine Rose schön ist, so prädiziert sie das Schön-Sein einer Rose. ${ }^{28}$

Im Hinblick auf die in dieser Arbeit auszuweisenden emotiv-kognitiven Formen des Erlebens und der damit zusammenhängenden Interdependenz von Gedanken und emotionalem Erleben ist auch die Konzeption propositionaler Einstellungen (hoffen, dass; befürchten, dass etc.) zu beachten. So sind bspw. der Wunsch oder die Hoffnung, dass es morgen doch Schneien möge, mögliche Einstellungen oder Bezogenheiten gegenüber der Proposition, dass es morgen Schneit. ${ }^{29}$ Demnach können auch die Gehalte von emotionsrelevanten Gedanken wie >es macht mich traurig, dass sie das gesagt hat< als propositionale Einstellungen betrachtet werden. ${ }^{30}$ So stellt Guttenplan fest: »[...] attitudes have contents and it is natural to regard these contents as propositions « ${ }^{31}$ So gesehen, können propositionale Einstellungen als mentale Zustände bzw. als (emotionsbezogene) Gedanken betrachtet werden: »[...] it is usual to regard

(einzelne) Gedanken keine Propositionen sein, weil sie als Token bzw. als konkretes Ereignis nicht falsch sein können:»Propositions, we shall say, are the sharable objects of the attitudes and the primary bearers of truth and falsity. This stipulation rules out certain candidates for propositions, including thought- and utterance-tokens, which presumably are not sharable, and concrete events or facts, which presumably cannot be false «. McGrath, Matthew: »Propositions«, in: The Stanford Encyclopedia of Philosophy, 2013, Online: http://plato.stanford.edu/archives/spr2o13/entries/propositions/ [zuletzt geprüft am 28.o9.2018], Abschnitt einleitender Erläuterungen.

28 Vgl. Soames, Scott: »Propositions«, in: The Routledge companion to philosophy of language, Russell, Gillian / Fara, Delia Graff [Hrsg.], New York: Routledge \& Kegan Paul 2012, S. 216.

29 Vgl. Levin, Janet: »Functionalism «, in: The Stanford Encyclopedia of Philosophy, 2013, Online: http://plato.stanford.edu/archives/fall2o13/entries/functionalism/ [zuletzt geprüft am 28.o9.2018], Abschnitt 4.3.

30 Mehr über propositionale Einstellungen vgl. z.B. Baker, Lynne R.: »Propositional Attitudes«, in: A companion to the philosophy of mind, Guttenplan, Samuel [Hrsg.], Malden, Massachusetts: Blackwell 2005. Oder: McKay, Thomas / Nelson, Michael: »Propositional Attitude Reports«, in: The Stanford Encyclopedia of Philosophy, 2013, Online: http:/plato.stanford.edu/archives/spr2o13/entries/prop-attitude-reports/ [zuletzt geprüft am 28.o9.2018]. Oder: Oppy, Graham: »Propositional attitudes«, 1998, Online: https://www.rep.routledge.com/articles/thematic/propositional-attitudes/v-1 [zuletzt geprüft am 25.09.2018]. Oder: Shier, David: »Propositional Attitude Reports«, in: The Routledge companion to philosophy of language, Russell, Gillian / Fara, Delia Graff [Hrsg.], New York: Routledge \& Kegan Paul 2012, S. 795-808. Oder: Schiffer, Stephen: »Thought and Language«, in: A companion to the philosophy of mind, Guttenplan, Samuel [Hrsg.], Malden, Massachusetts: Blackwell 2005, S. 589-594. Oder: Soames, Scott: »Propositions «, in: The Routledge companion to philosophy of language, Russell, Gillian / Fara, Delia Graff [Hrsg.], New York: Routledge \& Kegan Paul 2012, S. 209-220.

31 Guttenplan, Samuel:»Proposition«, in:A companion to the philosophy of mind, Guttenplan, Samuel [Hrsg.], Malden, Massachusetts: Blackwell 2005, S. 487. 
the attitudes as mental states directed either toward a sentence that expresses a proposition, or toward a proposition itself «. 32

Im Sinne einer vorläufigen phänomenologischen Arbeitsdefinition lässt sich mit Tye das Erleben nicht-emotiver Formen des Denkens insofern beschreiben, als mit Gedanken oft verbale Vorstellungen einhergehen, die neben den syntaktischen auch phonologische Strukturen unserer Muttersprache aufweisen. ${ }^{33}$ Denken, wenn es bewusst und mithin relevant für die hier vorgenommene Revision eines herkömmlichen Qualia-Begriffs ist, kann auch mit Vocate $^{34}$ bzw. Merleau-Ponty ${ }^{35}$ mit einem >inneren< Sprechen oder einer Vorstellung von Worten verglichen werden. Gedanken bestehen, so können wir mit diesen Ansätzen behaupten, nicht nur aus begrifflichen Gehalten im Sinne von Propositionen, ${ }^{36}$ sondern sie beinhalten auch vorgestellte auditive ${ }^{37}$ bzw. diverse andere sinnlich-imaginierte Erlebnisanteile. ${ }^{38}$

Eine Phänomenologie des Erlebens nicht-emotiver Formen des Denkens bzw. der Kognition, die sich nicht in erster Linie auf sinnlich-imaginierte

32 Ebd. [Hervorhebung E. E.].

33 Vgl. Tye, Michael: »Das Problem primitiver Bewusstseinsformen: Haben Bienen Empfindungen? «, in: Bewusstsein und Repräsentation, Esken, Frank / Heckmann, HeinzDieter [Hrsg.], Paderborn: Mentis [zuerst 1997, engl.]/1999, S. 94.

34 Vgl. Vocate, Donna R.: »Self-Talk and inner Speech: Understanding the Uniquely Human Aspects of Intrapersonal Communication «, in: Intrapersonal communication. Different voices, different minds, Vocate, Donna R. [Hrsg.], Hillsdale, New Jersey: Erlbaum 2009, S. 18. Vocate geht jedoch davon aus, dass Gedanken und »inner speech« nicht zwingend identisch sein müssen. Vgl. a.a.O., S. 17-18.

35 Vgl. dazu Merleau-Ponty, Maurice: Phänomenologie der Wahrnehmung, Berlin (West): De Gruyter [zuerst 1966, franz.]/1974, S. 211.

36 Vgl. dazu Guttenplan, Samuel [Hrsg.]: A companion to the philosophy of mind, Malden, Massachusetts: Blackwell 2005, S. 487. Vgl. ebenfalls Oppy, Graham: »Propositional attitudes«, 1998, Online: https://www.rep.routledge.com/articles/thematic/propositionalattitudes/v-1 [zuletzt geprüft am 25.09.2018].

37 Zur Konzeption von Denken als eine Art sinnere Stimmer vgl. auch Perry, John: "Subjectivity«, in: The Oxford Handbook of Philosophy of Mind, McLaughlin, Brian P. I Beckermann, Ansgar / Walter, Sven [Hrsg.], Oxford: Oxford University Press 2009, S. 226. Vgl. ebenfalls Levine, Joseph: »On Leaving Out What It's Like«, in: The nature of consciousness. Philosophical debates, Block, Ned J. [Hrsg.], Cambridge, Massachusetts: MIT Press 1997, S. 554.

38 Vgl. Damasio, Antonio R.: Descartes' error. Emotion, reason and the human brain, London: Vintage [zuerst 1994]/2006, S. 97, 106, 170 und 180. 
phänomenale Aspekte beruft, lässt sich bspw. mit Langsam, ${ }^{39}$ Searle, ${ }^{40} \mathrm{Jacob}^{41}$ oder Pitt ${ }^{42}$ deshalb postulieren, weil es irgendwie ist, Gedanken mit einem ganz bestimmten Gehalt zu haben, was für ein eigenes Qualia-Genre bzw. einer $»$ phenomenology of $\operatorname{cognition} \ll^{43}$ spricht. Im Hinblick auf weitere Formen nicht-emotiver Erlebnisweisen von kognitiven Vorgängen, soll diese phänomenologische Arbeitsdefinition insofern erweitert werden, als dem Verstehen (im Sinne eines »understanding-experience «) ${ }^{44}$ ebenfalls bestimmte, von vorgestellten Sinnesanteilen unabhängige phänomenale Eigenschaften zugesprochen werden können. ${ }^{45}$

Für eine phänomenologische Arbeitsdefinition von emotiven Formen des Denkens lassen sich diese als bewusste Zustände beschreiben, bei welchen bestimmte, durch Emotionen gefärbte Modi des Denkens erlebt werden. So ist es irgendwie, mit Sorge oder Freude an etwas zu denken und aufgrund dieses emotionalen Fokus' zu erleben, dass sich unsere Kognition in »affektspezifischen Fühl- und Denkbahnen $\ll^{46}$ bewegt. Die durch Emotionen gefärbten Denkmodi oder »Affektlogik[en $] \ll, 47$ wie z.B. die »Trauerlogik«, ${ }^{48}$ erstrecken sich eher über längere Zeiträume und können daher auch als typische phänomenale Gegebenheitsweisen von Stimmungen gesehen werden. So

39 Langsam, Harold L.: »Experiences, Thoughts, and Qualia«, Philosophical Studies, [2000] Vol. 99, No. 3, S. 272.

40 Vgl dazu auch Searle nach dem es für uns irgendwie ist, wenn wir etwas bewusst denken: »Is there something it is like, or feels like, just to sit and consciously think that $2+3=5$ ? [...] There is indeed something that it is like, or feels like, to think these things [...] «. Searle, John R.: The mystery of consciousness, New York: New York Review Books 1997, S. 201.

41 Jacob, Pierre: »Intentionality«, in: The Stanford Encyclopedia of Philosophy, 2010, Online: http://plato.stanford.edu/archives/fall201o/entries/intentionality/ [zuletzt geprüft am 28.09.2018], Abschnitt 10.o.

42 Vgl. Pitt, David: »The Phenomenology of Cognition or what is it Like to Think that P? «, Philosophy and Phenomenological Research, [2004] Vol. 69, No. 1, S. 2.

43 Vgl. ebd.

44 Strawson, Galen: Mental reality, Cambridge, Massachusetts: MIT Press 1994, S. 8.

45 Vgl. dazu z.B. Soldati, Gianfranco: »Begriffliche Qualia. Zur Phänomenologie der Bedeutung «, in: Anatomie der Subjektivität. Bewusstsein, Selbstbewusstsein und Selbstgefühl, Grundmann, Thomas / Hofmann, Frank / Misselhorn, Catrin et al. [Hrsg.], Frankfurt am Main: Suhrkamp 2005, S. 146-147 und 149. Vgl. auch Strawson, Galen: Mental reality, Cambridge, Massachusetts: MIT Press 1994, S. 12.

46 Ciompi, Luc: Die emotionalen Grundlagen des Denkens. Entwurf einer fraktalen Affektlogik, Göttingen: Vandenhoeck \& Ruprecht 1997, S. 182.

47 A.a.O., S. 45 .

48 A.a.O., S. $187-19$ o. 
bringt Ciompi unsere »affektiv-kognitiven >Schienen $<$ und `Eigenwelten $«{ }^{49}$ in Verbindung mit einer »einheitliche[n] Grundstimmung «, 50 die - bspw. im Falle der »Freude- und Lustlogik « ${ }^{51}$ - alles, was uns begegnet, »[...] zu einem schönen und sozusagen lächelnden Ganzen zusammenbindet«.52

\subsection{Berücksichtigung theoretischer Ansätze}

Für die Ausarbeitung eines phänomenal adäquaten Qualia-Begriffs und dessen Erweiterung auf emotiv-somatische bzw. emotiv-kognitive Erlebnisqualitäten werden zum einen neuropsychologische Theorien bzw. Zugänge wie z.B. diejenigen von A. Damasio ${ }^{53}$ und J. LeDoux ${ }^{54}$ berücksichtigt. Zum anderen wird auf physiologische (James-Lange-Theorien), phänomenlogische, komponentenorientierte und kognitivistische Emotionstheorien zurückgegriffen. ${ }^{55}$ Emotionstheorien nach James-Lange gehen, vereinfacht gesagt davon aus, dass Emotionen unauflöslich an körperliche Vorgänge gebunden sind. Diese Emotionstheorien fussen wissenschaftshistorisch, einerseits auf dem Aufsatz What is an Emotion? von William James von $1884,{ }^{56}$ andererseits

49 A.a.O., S. 179.

$50 \quad$ A.a.O., S. 193.

51 A.a.O., S. 192.

52 A.a.O., S. 193. Das ist auch der Grund dafür, dass (wie oben erwähnt) die Phänomenalität von Stimmungen vorwiegend im Abschnitt 8.2.4 einbezogen wird.

53 Vgl. Damasio, Antonio R.: The feeling of what happens. Body, emotion and the making of consciousness, London: Vintage 200o. Vgl. ebenfalls Damasio, Antonio R.: Descartes' error. Emotion, reason and the human brain, London: Vintage [zuerst 1994]/20o6.

54 Vgl. LeDoux, Joseph E.: Das Netz der Gefühle. Wie Emotionen entstehen, München \& Wien: Hanser 1998.

55 Emotionstheoretisch relevant sind darüber hinaus narrative Ansätze, die Emotionen als thematisch und chronologisch strukturierte Komplexe verstehen und nach Goldie auch Komponenten körperlicher Empfindungen einschliessen. Vgl. Goldie, Peter: The emotions. A philosophical exploration, New York: Oxford University Press 2002. Narrative Emotionstheorien weisen jedoch nicht nur einen kompositionalen Ansatz auf, sondern versuchen darüber hinaus zu ergründen, wie die verschiedenen Bestandteile einer Emotion miteinander verbunden sind. Vgl. Voss, Christiane: Narrative Emotionen. Eine Untersuchung über Möglichkeiten und Grenzen philosophischer Emotionstheorien, Berlin: De Gruyter 2004. In phänomenologischer Hinsicht wenig relevant sind freilich verhaltenstheoretische Emotionstheorien, nach denen Emotionen nur (Dispositionen zu) willkürliche(n) Verhaltensweisen wie Flucht oder unwillkürliche(m) Ausdrucksverhalten wie Zittern sind. Vgl. Ryle, Gilbert: The concept of mind, London: Penguin Books 1963.

Vgl. James, William: »What Is an Emotion?«, Mind, [1884] Vol. 9, No. 34. 
gehen sie auf den dänischen Physiologen Carl Georg Lange zurück, der 1887 eine ähnliche Theorie ausgearbeitet hat. ${ }^{57}$ Der James-Lange-Ansatz wird bis heute von emotionstheoretischen und neurobiologischen Zugängen immer wieder aufgegriffen, sowohl in kritisierender wie auch in affirmativer Weise. ${ }^{58}$ Des Weiteren werden phänomenologische Ansätze einbezogen. Diese betrachten emotionales Erleben im Allgemeinen als eine eigene mentale Kategorie, die sich weder auf Wünsche noch auf andere kognitive Elemente reduzieren lässt. Als Kategorie sui generis können Emotionen auf Gedanken eine gleichsam »färbende «59 Wirkung haben. In diesem Sinne spricht Stocker von »emotional thoughts « ${ }^{60}$ oder Goldie von »thinking of with feeling «. 61 Emotionstheoretisch relevant sind diesbezüglich auch die vorwiegend in der empirischen Gefühlsforschung verbreiteten (Mehr-) Komponententheorien, nach welchen sich Gefühle bzw. emotionales Erleben meistens aus einem Komplex von physiologischen, kognitiven, voluntativen, behavioralen und anderen Komponenten zusammensetzen. ${ }^{62}$ Es liegt in der Natur von Komponententheorien, dass sie sich mit anderen Emotionstheorien überschneiden, so betrachtet bspw. eine abgeschwächte Variante des Kognitivismus die

57 Vgl. dazu Lange, Carl G. / Kurella, Hans: Ueber Gemüthsbewegungen. Eine psychophysiologische Studie, Leipzig: T. Thomas 1887.

58 Vgl. Hartmann, Martin: Gefühle. Wie die Wissenschaften sie erklären, Frankfurt am Main: Campus 2010, S. 37. Vgl. auch LeDoux, Joseph E.: Das Netz der Gefühle. Wie Emotionen entstehen, München \& Wien: Hanser 1998, S. 48 und 5o.

59 Hartmann, Martin: Gefühle. Wie die Wissenschaften sie erklären, Frankfurt am Main: Campus 2010, S. 84.

6o Stocker, Michael: »Emotional Thoughts«, American Philosophical Quarterly, [1987] Vol. 24, No. 1, S.6o. Vgl. ebenfalls Stocker, Michael / Hegeman, Elizabeth: Valuing emotions, Cambridge, Massachusetts: Cambridge University Press 20o1, S. 13 und 45.

61 Goldie, Peter: The emotions. A philosophical exploration, New York: Oxford University Press 2002, S. 58. Vgl. auch a.a.O., 59, 71 und 77.

62 Vgl. z.B. Schachter, Stanley / Singer, Jerome E.: »Cognitive, Social and Physiological Determinants of Emotional State«, Psychological Review, [1962] Vol. 69, No. 5, S. 379-399. Vgl. ebenfalls Goldie, Peter: The emotions. A philosophical exploration, New York: Oxford University Press 2002, S. 155. Oder: Schachter, Stanley: Emotion, obesity and crime, New York: Academic Press 1971. Oder: Kenny, Anthony J. P.: Action, emotion and will, London \& New York: Routledge \& Kegan Paul 1963. Oder: Ciompi, Luc: Die emotionalen Grundlagen des Denkens. Entwurfeiner fraktalen Affektlogik, Göttingen: Vandenhoeck \& Ruprecht 1997. Oder:Damasio, Antonio R.: Descartes' error. Emotion, reason and the human brain, London: Vintage [zuerst 1994]/2006. Oder: Lyons, William: Emotion, Cambridge, Massachusetts: Cambridge University Press 1980. Oder: Demmerling, Christoph / Landweer, Hilge: Philosophie der Gefühle. Von Achtung bis Zorn, Stuttgart: Metzler 2007, S. 3-4 und 18. 
Urteils-Komponente als konstitutiven Aspekt einer Emotion, zu der jedoch noch weitere Komponenten dazukommen können. ${ }^{63}$ Die in dieser Arbeit ebenfalls zu reflektierenden kognitivistischen Emotionstheorien gehen grundsätzlich von der Annahme aus, dass nicht primär Körperempfindungen, sondern Überzeugungen, Urteile und Denken die wesentlichen Bestandteile von Emotionen bilden: »Thought replaced feeling as the principal element in the general conception of emotion ${ }^{6}{ }^{64}$

Die Anwendung der von mir für einen erweiterten Qualia-Begriff berücksichtigten Zugänge und Theorien gestaltet sich folgendermassen: Im Hinblick auf die von mir postulierten emotiven Formen des Erlebens werde ich vorwiegend auf die eingangs erwähnten neuropsychologischen Ansätze und physiologischen James-Lange-Theorien zurückgreifen. Bezüglich emotivkognitiver Erlebniszustände und im Zusammenhang mit der Interdependenz von Emotionen und Denken, gilt es, zwei emotionstheoretische Aspekte zu reflektieren: (1.) Inwiefern wirken sich Gedanken auf emotive Zustände aus? (2.) Können sich emotive Zustände im Sinne einer semotiven Färbungr massgeblich auf Kognition und Denken (bzw. auf deren intentionalen Fokus) auswirken und diesen somit einen >qualitativen Gehalt verleihen? Für (1.) wird vorwiegend auf kognitivistische Emotionstheorien zurückgegriffen werden. Emotive Aspekte von Gedanken (2.) werden dagegen in der philosophischen Forschung mit Ausnahme der $e$ emotional thoughts $\ll^{65}$ von Stocker,

63 Vgl. a.a.O., S. 3 .

64 Deigh, John: »Cognitivism in the Theory of Emotions«, Ethics, [1994] Vol. 104, No. 4, S. 824. Vgl. z.B. ebenfalls Solomon, Robert C.: The passions, Notre Dame, Indiana: University of Notre Dame Press [zuerst 1976]/1983. Oder: Nussbaum, Martha C.: Upheavals of thought. The intelligence of emotions, Cambridge, Massachusetts: Cambridge University Press 20o1. Oder: Lyons, William: Emotion, Cambridge, Massachusetts: Cambridge University Press 1980. Oder: Sousa, Ronald de / [Übers.: Pape, Helmut]: Die Rationalität des Gefühls, Frankfurt am Main: Suhrkamp [zuerst 1987, engl.]/20o9, S. 21-50 und 281-311. Oder: Deigh, John: „Cognitivism in the Theory of Emotions«, Ethics, [1994] Vol. 104, No. 4, S. 824-854.

65 Vgl. Stocker, Michael: »Emotional Thoughts«, American Philosophical Quarterly, [1987] Vol. 24, No. 1. Vgl. dazu auch Stocker, Michael / Hegeman, Elizabeth: Valuing emotions, Cambridge, Massachusetts: Cambridge University Press 20o1. Vgl. ebenfalls Stocker, Michael: »Einige Betrachtungen zu intellektuellen Wünschen und Emotionen «, in:Philosophie der Gefühle, Döring, Sabine [Hrsg.], Frankfurt am Main: Suhrkamp [zuerst 2004, engl.]/20o9. 
der »emotive thoughts« von Pugmire ${ }^{66}$ bzw. des »thinking of with feeling « 67 von Goldie ${ }^{68}$ kaum berücksichtigt. ${ }^{69}$ Deshalb werden in dieser Arbeit auch psychologische Theorien (mit komponentenorientiertem Ansatz) zur Interdependenz von Denken und Emotionen (wie z.B. L. Ciompi, A. Ellis, und S. Schachter $)^{70}$ mit einbezogen.

Bezüglich der postulierten Phänomenalität der Intentionalität bzw. phänomenalen Aspekten der Intentionalität (von Emotionen) werde ich u.a. auf Überlegungen von Graham, ${ }^{71}$ Ciompi $^{72}$ und auf Goldies Konzeption des »feeling towards «73 zurückgreifen. Nicht zuletzt werde ich bezüglich der nachzuweisenden Phänomenalität nicht-emotiver Formen des Denkens

66 Bei Pugmire findet sich die Konzeption von »emotive thoughts«: »[...] thoughts lie at the heart of emotional arousal. In other words, perhaps emotional feelings themselves are thoughts, emotive thoughts [...]«. Pugmire, David: Rediscovering emotion, Edinburgh: Edinburgh University Press 1998, S. 63.

67 Diese emotiven Formen des Denkens lassen sich, mit Goldie auf eine kurze Formulierung gebracht, wie folgt beschreiben: »Feeling towards, as it is thinking of with feeling, is a sort of thinking of«. Goldie, Peter: The emotions. A philosophical exploration, New York: Oxford University Press 2002, S. 19.

68 Goldie spricht auch im Hinblick auf die Intentionalität von Emotionen von »thinking of with feeling«. A.a.O., S. 58. Vgl. auch a.a.O., S. 71 und 77.

69 Ebenfalls relevant für die Thematik der emotiven Aspekte von Gedanken sind die von Pugmire an anderer Stelle erwähnten »embodied appraisals«. Pugmire, David: »Emotionen und ihre empirische Untersuchung «, in: Philosophie der Gefühle, Döring, Sabine [Hrsg.], Frankfurt am Main: Suhrkamp [zuerst 2006, engl.]/20og, S. 332. Vgl. ebenfalls a.a.O., 346 .

70 Vgl. z.B. Ciompi, Luc: Die emotionalen Grundlagen des Denkens. Entwurf einer fraktalen Affektlogik, Göttingen: Vandenhoeck \& Ruprecht 1997. Oder: Ellis, Albert: Die rationalemotive Therapie. Das innere Selbstgespräch bei seelischen Problemen und seine Veränderung, München: J. Pfeiffer 1977, S. 29. Oder: Schachter, Stanley: Emotion, obesity and crime, New York: Academic Press 1971. Oder: Schachter, Stanley / Singer, Jerome E.: »Cognitive, Social and Physiological Determinants of Emotional State«, Psychological Review, [1962] Vol. 69, No. 5, S. 379-399.

71 Vgl. z.B. Graham, George / Horgan, Terence / Tienson, John: »Phenomenology, Intentionality, and the Unity of the Mind «, in: The Oxford Handbook of Philosophy of Mind, McLaughlin, Brian P. / Beckermann, Ansgar / Walter, Sven [Hrsg.], Oxford: Oxford University Press 2009, S. 512-537.

72 Vgl. z.B. Ciompi, Luc: Die emotionalen Grundlagen des Denkens. Entwurf einer fraktalen Affektlogik, Göttingen: Vandenhoeck \& Ruprecht 1997, S. 102 und 193.

73 Goldie, Peter: The emotions. A philosophical exploration, New York: Oxford University Press 2002, S. 57. 
Überlegungen von Chalmers, ${ }^{74}$ Perry, ${ }^{75}$ Searle, ${ }^{76} \mathrm{Jacob},{ }^{77}$ Graham et al., ${ }^{78} \mathrm{Kobes}^{79}$ und Pitt ${ }^{80}$ berücksichtigen ${ }^{81}$ sowie den kognitionswissenschaftlichen Zugang von Vocate. ${ }^{82}$ Ebenfalls werde ich auf Strawson, Jung, Heilinger und Soldati eingehen, für die der kognitive Vorgang des Verstehens eine phänomenale Qualität sui generis hat. ${ }^{83}$

74 Vgl. dazu Chalmers, David J.: The conscious mind. In search of a fundamental theory, New York: Oxford University Press 1996, S. 6 und 10.

75 Vgl. dazu Perry, John: »Subjectivity«, in: The Oxford Handbook of Philosophy of Mind, McLaughlin, Brian P. / Beckermann, Ansgar / Walter, Sven [Hrsg.], Oxford: Oxford University Press 2009, S. 224.

76 Vgl. dazu Searle, John R.: The mystery of consciousness, New York: New York Review Books 1997, S. 201.

77 Vgl. dazu Jacob, Pierre: ॥ntentionality«, in: The Stanford Encyclopedia of Philosophy, 2010, Online: http://plato.stanford.edu/archives/fall2o1o/entries/intentionality/ [zuletzt geprüft am 28.09.2018], Abschnitt 10.o.

78 Vgl. Graham, George / Horgan, Terence / Tienson, John: »Phenomenology, Intentionality, and the Unity of the Mind«, in: The Oxford Handbook of Philosophy of Mind, McLaughlin, Brian P. / Beckermann, Ansgar / Walter, Sven [Hrsg.], Oxford: Oxford University Press 2009, S. 513. Vgl. auch a.a.O., S. 516.

79 Vgl. Kobes, Bernard W.: »Access and what it is like«, Behavioral and Brain Sciences, [1995] Vol. 18, No. 2, S. 26o.

8o Vgl. Pitt, David: »The Phenomenology of Cognition or what is it Like to Think that P? «, Philosophy and Phenomenological Research, [2004] Vol. 69, No. 1, S. 2.

81 Diese Autoren gehen explizit davon aus, dass es irgendwie ist, Gedanken mit einem ganz bestimmten Gehalt zu haben.

82 Vgl. Vocate, Donna R.: »Self-Talk and inner Speech: Understanding the Uniquely Human Aspects of Intrapersonal Communication«, in: Intrapersonal communication. Different voices, different minds, Vocate, Donna R. [Hrsg.], Hillsdale, New Jersey: Erlbaum 2009.

83 Zur Konzeption der Phänomenalität des Verstehens vgl. Strawson, Galen: Mental reality, Cambridge, Massachusetts: MIT Press 1994. Oder: Jung, Matthias / Heilinger, Jan-Christoph [Hrsg.]: Funktionen des Erlebens. Neue Perspektiven des qualitativen Bewusstseins. Band 5: Humanprojekt, Berlin: De Gruyter 20og. Oder: Soldati, Gianfranco: »Begriffliche Qualia. Zur Phänomenologie der Bedeutung«, in: Anatomie der Subjektivität. Bewusstsein, Selbstbewusstsein und Selbstgefühl, Grundmann, Thomas / Hofmann, Frank / Misselhorn, Catrin et al. [Hrsg.], Frankfurt am Main: Suhrkamp 2005, S. 148. 